\title{
Analysis of SO/AO elastic wave mode conversion phenomenon in glass fibre reinforced polymers
}

\author{
Tomasz Wandowski ${ }^{1,},{ }^{*}$ Pawel Malinowski ${ }^{1}$, and Wieslaw Ostachowicz ${ }^{1}$ \\ ${ }^{1}$ Institute of Fluid-Flow Machinery, Polish Academy of Sciences, 80-231 Gdansk, Poland
}

\begin{abstract}
In this paper results of experimental analysis of elastic guided wave mode conversion phenomenon in glass fibre reinforced polymers GFRP are presented. Results of research presented in this paper are strictly focused on S0/A0' mode conversion caused by discontinuities in the form of circular teflon insert simulating damage and impact damage. Experimental research is based on non-contact elastic wave sensing with utilisation of scanning laser Doppler vibrometer SLDV and full wave-field measurements. In presented research influence of location of circular teflon insert on S0/A0' mode conversion is investigated. Teflon inserts were located between layers of composite material at different depth. Moreover influence of impact damage with different energy on S0/A0' mode conversion is investigated. Analysis of influence of investigated discontinuities on S0/A0' mode conversion are based on the elastic wave mode filtration in frequency-wavenumber domain. Mode filtration process allows to remove effects of unwanted type of mode propagation in forward or backward direction. Effects of S0/A0' mode conversion are characterise by mode conversion indicator based on amplitude of new mode A0'.
\end{abstract}

\section{Introduction}

Structural Health Monitoring (SHM) is more and more attracted attention of many researchers. Application of SHM systems allows to reduce the number of unexpected maintenance and service operation related to structural degradation of exploited structure. Among many diagnostic techniques that could be utilized in SHM very popular is guided wave propagation method. It is based on propagation of elastic waves that are guided in structural elements. Initiation of the damage is related to change of physical properties like for example stiffness reduction which changes then the elastic wave propagation. In this method very often two fundamental modes of elastic guided waves are utilized: symmetric S0 and anti-symmetric A0 [1]. Different effect related to interaction of wave with discontinuities are investigated: wave reflection, wave scattering, change of wavelength or mode conversion.

Research presented in this paper is related to analysis of influence of discontinuities on mode conversion phenomenon. There is still limited number of works related to the analysis of elastic waves mode conversion phenomenon especially for composite structures.

\footnotetext{
*Corresponding author: tomaszw@imp.gda.pl
} 
This topic was investigated by authors of work [2] where the effect of continuous mode conversion in CFRP plate were analyzed. In the paper [3] mode conversion phenomenon and continuous wavelet transform were utilized for damage localisation in sandwich structures. Authors of paper [4] utilized mode conversion phenomenon for estimation of rectangular notch parameters in the metallic plate. In the research work [5] phenomenon of Rayleigh wave to Lamb wave mode conversion was investigated. Authors presented numerical and experimental results for the metal plate. Authors of paper [6] deal with the problem of shear horizontal $(\mathrm{SH})$ mode conversion in the tapered aluminium plate. In work [7] several comparison experiments were conducted to compare the efficiency of mode conversion phenomenon in the pipeline. Authors of paper [8] utilized the 3D scanning laser vibrometry and mode conversion effect for damage detection. In this research mode conversion effect was utilized as an indicator of damage. Authors of work [9] analyzed the mode conversion phenomenon for teflon insert located symmetrically in respect to the thickness of composite panels. In numerical results there was no mode conversion for discontinuity located symmetrically in respect to the thickness of composite panel. However, experimental results presented in this paper indicated that in the case of teflon insert symmetrically located (the same number of plies above and below the insert) mode conversion S0/A0' was noticed. It proved that perfect symmetry is not achieved in real conditions.

Signal processing for full wavefield elastic wave based damage detection could be based on analysis in frequency-wavenumber domain [10]. Authors of paper [11] proposed application of scanning spatial-wavenumber filter for damage detection in composite aerospace structure. Signal analysis in frequency-wavenumber domain could be especially useful for investigations of mode conversion effects. Authors of works [9], [12], [13] utilized frequency-wavenumber processing for modes filtration. This approach could remove propagation of unwanted mode from the wavefield.

In presented research influence of location (in respect to the sample thickness) of circular teflon insert on S0/A0' mode conversion is investigated. Teflon inserts were located between layers of composite material at different depth. Moreover influence of impact damage with different energies on $\mathrm{S} 0 / \mathrm{A} 0$ ' mode conversion is investigated.

\section{Measurement set-up}

Experimental set-up consisted of two investigated composite samples and Polytec scanning laser vibrometer PSV-400. Investigated samples were made out of glass fibre reinforced polymer panels. Dimensions of the panels were following: $500 \mathrm{~mm}$ x $500 \mathrm{~mm}$ and thickness $\sim 1.5 \mathrm{~mm}$. Each investigated sample consisted of twelve layers of VV192T/202 IMP503 prepregs with orientations: [0/90/0/90/0/90]s. Two samples were investigated in this study. First sample contained discontinuities in the form of four circular teflon inserts with diameter $20 \mathrm{~mm}$. Teflon inserts were located between different layers at different depth (Fig. 1). Second sample contained discontinuities in the form of impact damage with energies $5 \mathrm{~J}, 10 \mathrm{~J}$ and $15 \mathrm{~J}$ (Fig. 2). Impact damage was created by steel ball dropped from selected height in order to achieve desired impact energy level. Each sample was equipment with piezoelectric transducer utilized for elastic wave excitation. Piezoelectric transducer was placed at the middle of the sample (Fig. 1, Fig. 2). Excitation signal was in the form of five cycles of sine modulated by Hann window. Frequency of excitation was equal $200 \mathrm{kHz}$. Elastic wave sensing was conducted in non-contact way using laser vibrometer. Measurements were taken at opposite side to the piezoelectric transducer (bottom surface in Fig. 1, Fig. 2). It needs to be underlined here, that only one scanning laser head of laser vibrometer was utilized - measurements were taken along laser beam approximating out-of-plane guided elastic wave particle velocities. 

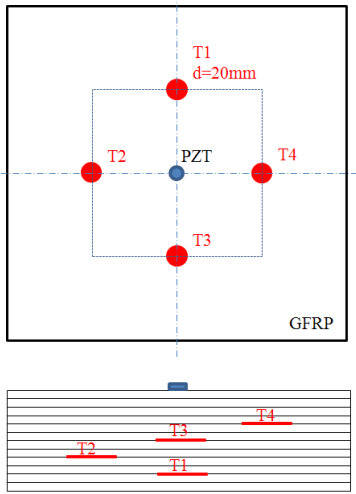

Fig. 1. GFRP sample no. 1 with teflon inserts.

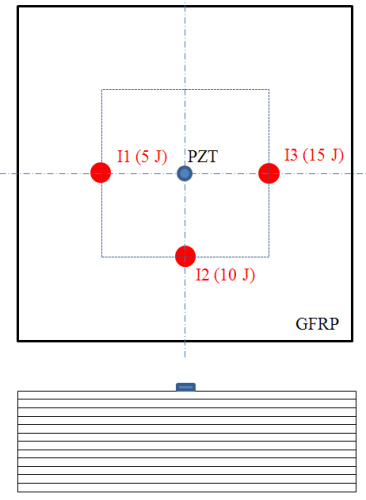

Fig. 2. GFRP sample no. 2 with impact damage.

\section{Analysis of full wavefield}

\subsection{Time-space domain}

Wave excitation was conducted on the top surface of sample by piezoelectric transducer. Measurements of elastic wave propagation were taken for the dense square grid of points (333 by 333) on the bottom surface of sample. As result of measurement 3D matrix of signals $s(x, y, t)$ was obtained. This matrix contains signals in time domain acquired from measurement grid. Collected data has a form of wavefield images of propagating elastic waves at the bottom surface of the plate. In the Fig. 3a) result in the form of full wavefield pattern for the GFRP sample no. 1 with circular teflon inserts (see Fig. 1) is presented. Elastic waves are excited in the point located in the middle of the sample. Excitation frequency $(200 \mathrm{kHz})$ was selected in the way that only fundamental modes symmetric $\mathrm{S} 0$ and antisymmetric $\mathrm{A} 0$ propagate.

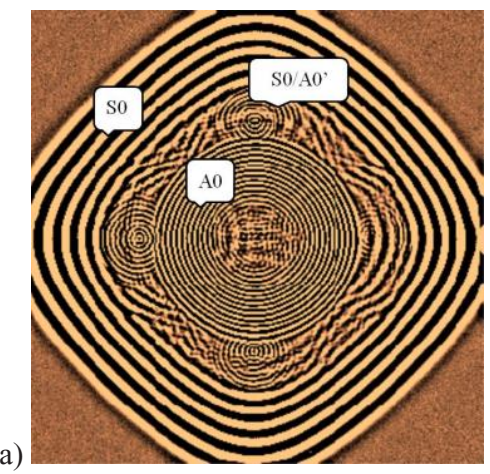

b)

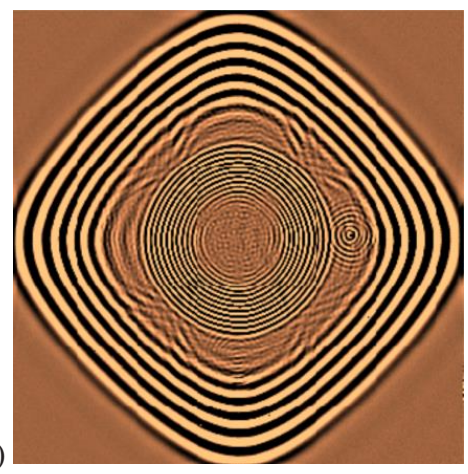

Fig. 3. Full wavefield patterns at selected time instances for: a) sample no. 1 with teflon inserts, b) sample no. 2 with impact damage.

Two modes can be distinguished due to the fact that they have different wavelength ( $\mathrm{S} 0$ has longer wavelength than $\mathrm{A} 0$ ) and velocity of propagation ( $\mathrm{S} 0$ is faster than $\mathrm{A} 0$ ). Moreover effects of mode conversion S0/A0' are observed. In the location of circular teflon inserts symmetric S0 mode is converted to fundamental antisymmetric mode denoted A0'. Intensity of mode conversion could be characterise by amplitude of $\mathrm{A} 0$ ' mode generated as a result of conversion of $\mathrm{S} 0$ mode. 
Analysing results presented in Fig. 3a) it can be noticed that amplitude of A0' mode depends on the depth at which teflon insert was located (compare with Fig. 1). Largest amplitude of A0' mode was achieved in the case of teflon insert T1 (Fig. 1).

In the Fig. 3b) result in the form of full wavefield pattern for GFRP sample no. 2 including impact damage is presented. In this case propagation of fundamental S0 and A0 mode is also observed. Moreover, S0/A0' mode conversion at location of impact damage with energies $10 \mathrm{~J}$ and $15 \mathrm{~J}$ is observed. Amplitude of A0' mode is higher for impact damage with higher energy $(15 \mathrm{~J})$. Impacts were made out on the top surface of sample.

\subsection{Frequency-wavenumber domain}

In next step one dimensional analysis of time signals in frequency-wave number domain was conducted. In this purpose four sets of time-space domain signals were selected. For this kind of analysis only signals gathered along four lines running from excitation point (piezoelectric transducer) through investigated discontinuities to the edges of plate are selected. In the Fig. 5 these lines (L1-L4) for the sample no. 1 with teflon inserts are presented. Depending on the direction of line sets of time-space signals $s(x, t)$ or $s(y, t)$ are obtained. The set of continuous signals in the time-space $s(x, t)$ domain $s(x, t)$ could be transformed to frequency-wavenumber $\left(k_{x}, f\right)$ domain using 2D Fast Fourier Transform FFT defined as follows:

$$
s\left(k_{x}, f\right)=\int_{-\infty-\infty}^{\infty} \int_{-\infty}^{\infty} s(x, t) \cdot e^{-j\left(2 \pi f t-k_{x} x\right)} d x d t
$$

where: $k_{x}$-wavenumber along x direction, $f$ - frequency. Such same transformation can be made for signals set $s(y, t) s(y, t)$ taken for line along $y$ direction. Result of such a transform for signals set for line L1 in sample no. 1 with teflon insert T1 is presented in Fig. 5.

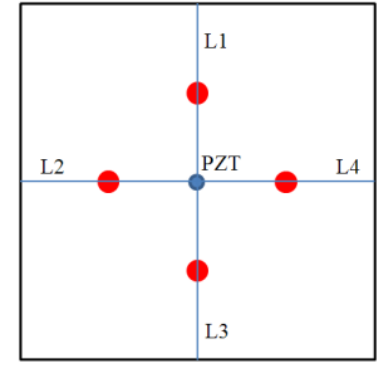

Fig. 4. Locations of lines L1-L4 for signal analysis in frequency-wavenumber domain.

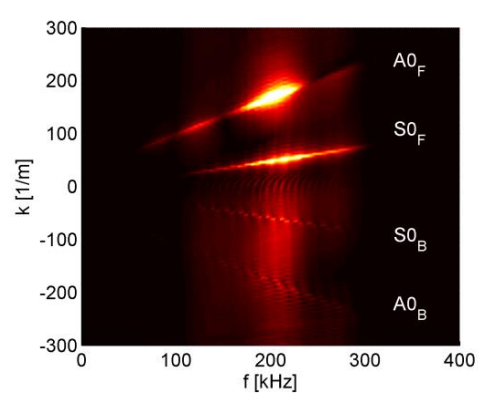

Fig. 5. Signals in frequency-wavenumber domain for teflon insert T1 in sample no. 1 
In Fig. 5 wavenumbers related to forward propagation of symmetric $\mathrm{S}_{\mathrm{F}}$ and antisymmetric $\mathrm{A} 0_{\mathrm{F}}$ modes are visible. Moreover, wavenumbers releated to backward propagation of symmetric and antisymmetric modes $\mathrm{S}_{\mathrm{B}}$ and $\mathrm{A} 0_{\mathrm{B}}$ are visible. Having representation of full wavefield in the frequency-wavenumber domain it is possible to conduct the process of mode filtration. It is possible to remove whole propagation of selected mode or only direction of propagation (forward or backward) of chosen mode using specially designed mask of the filter. After application of filter mask for frequencywavenumber data inverse 2D Fourier transform is utilized to transform filtered wavefield back to time-space domain. More information about this method could be found in papers [12], [13].

In the Fig. 6a) time-space signal gathered for sample no. 1 along line L1 is presented. This signal was plotted for the time instant at which propagation of A0 and S0 mode is clearly vissible. Moreover, at this time instant S0 mode already reached the location of teflon insert $\mathrm{T} 1$ indicated by to vertical lines.
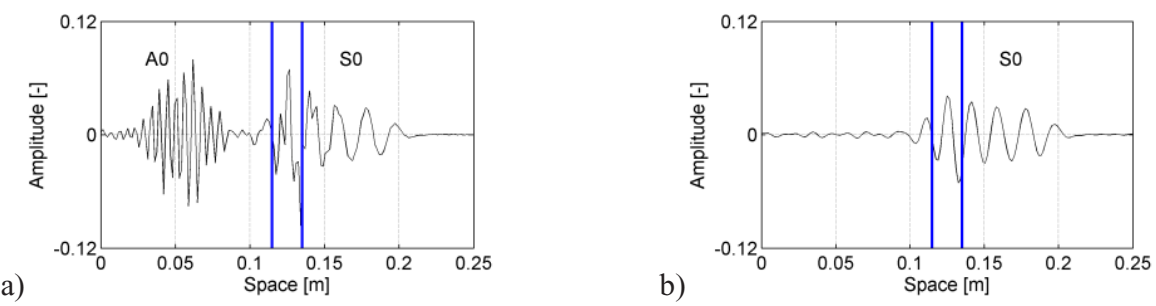

Fig. 6. Signals in time-space domain: a) without mode filtration, b) mode filtration - only propagation of S0 mode.

Effect of S0/A0' mode conversion could be noticed analysing the non-regular shape of S0 mode in Fig. 6a). Next after the mode filtration process forward propagation of A0 mode $\left(\mathrm{A} 0_{\mathrm{F}}\right)$ is removed. As result of this process only propagation of $\mathrm{S} 0$ mode is visible (Fig. $6 b)$ ). Mode filtration process could be helpful in the analysis of mode conversion.

\section{Analysis of SO/A0' mode conversion}

Mode filtration process was utilized in order to analyse S0/A0 mode conversion on teflon inserts and impact damage. S0/A0' mode conversion could be clearly observed after filtration (removing) of forward propagation of $\mathrm{S}_{\mathrm{F}}$ modes and backward propagation of $\mathrm{A} 0_{\mathrm{B}}$ and $\mathrm{S}_{\mathrm{B}}$. Only forward propagation of $\mathrm{A} 0_{\mathrm{F}}$ mode is visible. Additionally time instant need to be selected in such a way that S0 mode already reach the location of discontinuity, like teflon insert in analysed case (Fig. 6b)). In this case in the location of teflon insert newly created (after conversion) and forward propagating mode A0' could be observed.

S0/A0' mode conversion could be also visible after the filtration of forward propagating modes $\mathrm{S}_{\mathrm{F}}$ and $\mathrm{A} 0_{\mathrm{F}}$, and backward propagation of $\mathrm{S}_{\mathrm{B}}$. In this case only backward propagation of $\mathrm{A} 0_{\mathrm{B}}$ is observed where at the location of insert backward propagating mode A0' could be observed.

\subsection{Sample with teflon inserts}

In the Fig. 7 selected signals in time-space domain for sample no. 1 with teflon inserts (Fig. 1) were presented. There are signals after mode filtration process and containing only forward propagation of A0 mode. These signals are for the time instant at which mode S0 (in wavefield without mode filtration) reached the location of teflon insert (see Fig. 6b)).

In the Fig. 7a) forward propagation of A0 mode excited by piezoelectric transducer is visible. Moreover, forward propagation of A0' mode resulting from S0 mode conversion at 
location of teflon insert $\mathrm{T} 1$ is visible. Teflon insert location is indicated by vertical lines. In the Fig. 7b) also forward propagation of A0 mode and A0' modes is visible. A0' mode was generated after conversion of S0 mode at teflon insert T3. Amplitude of mode A0' is higher in the case of teflon insert $\mathrm{T} 1$ which is located closer to the surface of the sample measured with vibrometer.

In the Fig. 8 time-space signals for the same sample were presented. In this case only backward propagation of A0 mode is visible. The time instant is the same like in result presented in Fig. 7. In Fig. 8a) propagation of mode A0' (generated after conversion) could be only observed. Source of conversion was teflon insert T1. This is because the fact that forward propagation of A0 mode was removed by mode filtration process. There is no reflection of A0 mode from edge of plate because at this time instant $\mathrm{A} 0$ mode even have not reached yet location of teflon insert (compare with Fig. 7). In Fig. 8b) backward propagation of A0' mode can be noticed. This is mode generated as result of interaction of S0 mode with teflon insert T3. Comparing amplitudes of A0' mode for teflon insert T1 and T3, it is clearly seen that larger amplitude was achieved in the case of insert T1 located closer to the surface where the measurements were taken.

a)

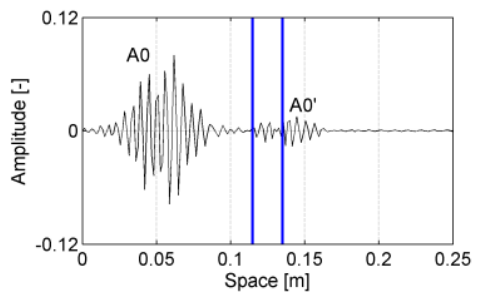

b)

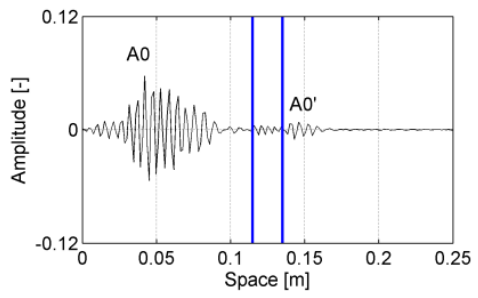

Fig. 7. Forward propagation of $\mathrm{A} 0$ and $\mathrm{A} 0$ ' mode, teflon insert : a) T1, b) T3. a)

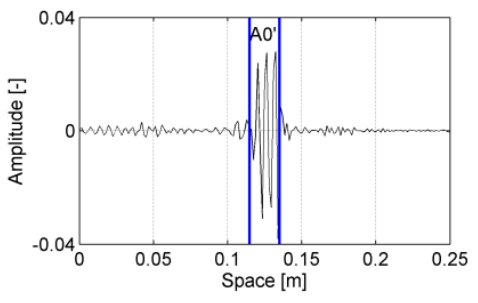

b)

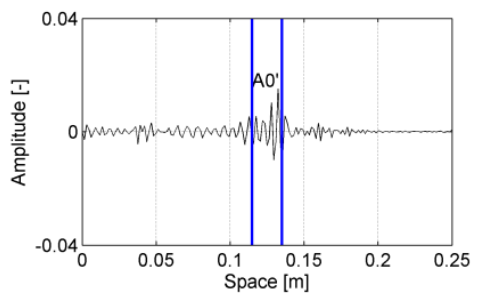

Fig. 8. Backward propagation of $\mathrm{A} 0$ ' mode, teflon insert : a) T1, b) T3.

The same analysis were conducted for teflon inserts T2 and T4. Based on this analysis mode conversion index $\mathrm{MCl}$ was formulated. It compare amplitudes of S0 mode reaching teflon insert with the amplitude of A0' mode generated as result of conversion of S0 mode. In this case amplitude of backward propagated A0' mode is taken for consideration. This index is formulated as follows:

$$
\mathrm{MCl}=\frac{A 0^{\prime}}{S O} C l=\frac{A 0 r}{S O},
$$

where: $\mathrm{MCl}$ - conversion index, $A 0^{\prime} A 0^{\prime}$ - amplitude of new mode (backward propagating) resulting from conversion of S0 mode, $S 0$ - amplitude of S0 mode utilized for normalisation. It allows to eliminate the problem of different amplitudes of S0 mode in different directions.

Table 1. $M C I$ values for teflon inserts at different depth.

\begin{tabular}{|c|c|c|}
\hline Discontinuity & $\begin{array}{c}\text { Layers } \\
\text { (from the bottom) }\end{array}$ & MCI \\
\hline
\end{tabular}




\begin{tabular}{|c|c|c|}
\hline teflon insert T1 & $2 / 3$ & 0,192 \\
\hline teflon insert T2 & $4 / 5$ & 0,109 \\
\hline teflon insert T3 & $6 / 7$ & 0,055 \\
\hline teflon insert T4 & $8 / 9$ & 0,051 \\
\hline
\end{tabular}

Amplitudes of both modes are taken at the time instant of observed mode conversion. Table 1 contains values of conversion index $\mathrm{MCl}$ for teflon inserts T1-T4. Largest value was achieved for teflon insert T1 located closest to the surface of sample where measurements were conducted. The lowest value of conversion index was achieved for teflon insert T4 located deepest in the sample (closer to the surface where wave were excited). Conversion index values decrease with increasing depth of teflon from T1 to T4.

\subsection{Sample with impact damage}

Next, similar analysis was performed for sample no. 2 with impact damage (Fig. 2). In the Fig. 9 time-space signals presenting only forward propagation of A0 mode are presented. Signal presented in Fig. 9a) is related to propagation along line L1 without impact damage (compare Fig. 2 and Fig. 4). In this figure propagation of A0 mode excited by piezoelectric transducer is solely visible.

a)

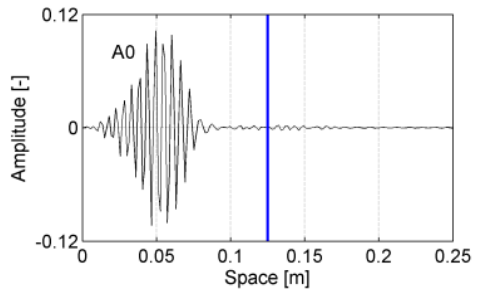

b)

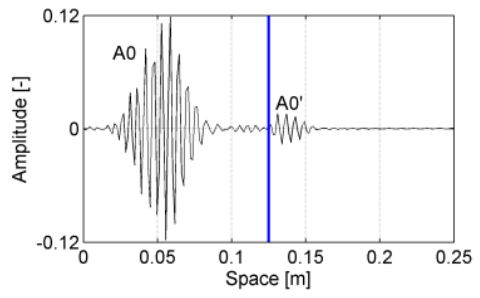

c)

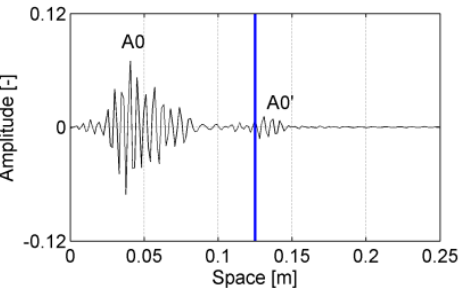

a)

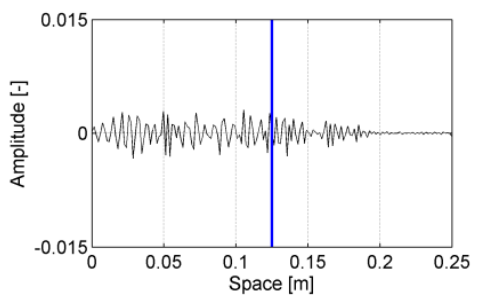

b)

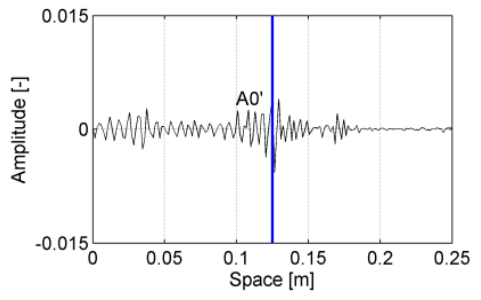

c)

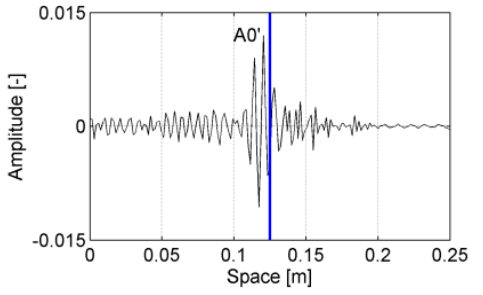

Fig. 9. Forward propagation of $\mathrm{A} 0$ and $\mathrm{A} 0$ ' mode, for : a) referential case, b) impact $10 \mathrm{~J}$, c) impact $15 \mathrm{~J}$.
Fig. 10. Backward propagation of $\mathrm{A} 0$ ' mode, for: a) referential case, b) impact $10 \mathrm{~J}$, c) impact $15 \mathrm{~J}$.

In the Fig. 9b) signal taken along line L3 (see Fig. 2 and Fig. 4) for impact damage with energy $10 \mathrm{~J}$ was presented. In this case also forward propagation of excited A0 mode is observed. Beside this, additionally A0'mode resulting from S0/A0' mode conversion is also 
visible. The same results were obtained for signal taken along line L4 for impact damage with energy $15 \mathrm{~J}$ (Fig. 9c)).

In next step analysis of backward propagation of A0 mode was conducted. Results of this analysis were presented in Fig. 10. Result presented in Fig. 10a) is related to wave propagation through the line L1 without any damage. There is only noises visible without any S0/A0 mode conversion. Next, in the Fig. 10b) signal taken along line L3 is presented. Here the effect of $\mathrm{S} 0$ mode conversion at the location of impact damage with energy $10 \mathrm{~J}$ is represented by A0' mode. In this case amplitude of A0' is larger than noises in Fig. 10a). Similar situation is presented in the Fig. 10c) where A0' mode as result of conversion of S0 mode at impact damage with energy $15 \mathrm{~J}$ located at line L4 is visible. In this case amplitude of A0' mode is larger than in the case of impact energy $10 \mathrm{~J}$.

In order to compare results, mode conversion index $\mathrm{MCl}$ was utilized. In the table 2 results in the form of mode conversion index for: referential case (without damage) and for impact damage with energies $5 \mathrm{~J}, 10 \mathrm{~J}$ and $15 \mathrm{~J}$ were presented. This index was calculated based on amplitude of backward propagated A0' mode. Smallest value of conversion index was achieved for referential case. In this case amplitude of noise in the signal at the same distance from excitation point as for the impact damage was taken for the analysis. Larger values of $\mathrm{MCl}$ were achieved for the cases of impact damage with energies $5 \mathrm{~J}$ and $10 \mathrm{~J}$ respectively. Largest values of $\mathrm{MCl}$ was obtained for the impact energy $15 \mathrm{~J}$.

Table 2. $M C I$ values for impact damage with different energies.

\begin{tabular}{|c|c|}
\hline Discontinuity & MCI \\
\hline referential & 0,129 \\
\hline impact 5 J & 0,317 \\
\hline impact 10 J & 0,437 \\
\hline impact 15 J & 0,608 \\
\hline
\end{tabular}

It should be underlined that only impact damage with energies $10 \mathrm{~J}$ and $15 \mathrm{~J}$ were clearly detected. In the case of impact damage with energy $5 \mathrm{~J}$ there was no mode conversion effect visible in the full wavefield pattern in Fig. 3b). Moreover, there is hard to distinguish referential state and impact with energy $5 \mathrm{~J}$ in the time-space mode filtered signals. Differences are clearly visible for higher energies of impacts: $10 \mathrm{~J}$ and $15 \mathrm{~J}$ (Fig. $10 \mathrm{~b}, \mathrm{c})$ ).

\section{Conclusions}

Results of conducted research indicate that teflon inserts and impact damage are sources of S0/A0' mode conversion. Smallest detectable impact damage based on MCI has energy $5 \mathrm{~J}$ and $10 \mathrm{~J}$ if we compare the time-space signals. Amplitude of A0' mode depends on the dept at which teflon insert is located. Amplitude of A0' mode is also related to impact energy. Depth of teflon insert and extent of impact damage can be characterise by utilisation of mode conversion index $\mathrm{Cl}$. Obtained results should be validated in larger set of samples and cases.

Authors would like to gratefully acknowledge financial support given by Polish National Science Centre under grant agreement no. UMO-2014/13/D/ST8/03167 in the frame of SONATA project entitled: "Investigation of elastic wave mode conversion phenomenon in thin-walled structures with discontinuities".

\section{References}


1. V. Giurgiutiu, Ultrasonics, 48 (2), (2008)

2. B. Hennings, R. Lammering, Composite Structures, 151, 142-148, (2016)

3. S.M.H. Hosseini, S. Duczek, U. Gabbert, J Nondestruct Eval 33:152-165 (2014)

4. A. Ghadami, M. Behzad, et al., Archive of Appl. Mech., ISSN 0939-1533 (2015)

5. C. Schaal, H. Samajder, H. Baid, A. Mal, J of Sound and Vibr. 353, 150-163 (2015)

6. Nurmalia, N. Nakamura, H. Ogi, M. Hirao, et al., NDT \& E Internat,45, 1 (2012)

7. F. Sun, Z. Sun, Q. Chen, R. Murayama, H. Nishino, Sensors (Basel) 16, 10 (2016)

8. L. Pieczonka, L. Ambrozinski, et al., Optics and lasers in enginer. 99, 80-87 (2017)

9. T. Wandowski, P. Malinowski, P. Kudela, et al, Proc. of SPIE 1060028, (2018)

10. P.D. Juarez, C. Leckey, Ultrasonics 62, (2015)

11. Y. Ren, L. Qiu, S. Yuan, Z. Su, Mechanical Systems and Signal Processing 90, (2017)

12. M. Ruzzene, Smart Materials and Structures 16, 6 (2007)

13. Z. Tian, L. Yu, Journal of Intelligent Material Systems and Structures 25, 9 (2014) 